RESEARCH BRIEF

\title{
Public Awareness of Health-Related Risks From Uncontrolled Hypertension
}

\author{
Rahoul Ahuja, MD ${ }^{1,2}$; Carma Ayala, PhD, MPH, RN ${ }^{1}$; Xin Tong, $\mathrm{MPH}^{1}$; \\ Hilary K. Wall, $\mathrm{MPH}^{1}$; Jing Fang, $\mathrm{MD}, \mathrm{PhD}^{1}$
}

Accessible Version: www.cdc.gov/pcd/issues/2018/17_0362.htm

Suggested citation for this article: Ahuja R, Ayala C, Tong X, Wall HK, Fang J. Public Awareness of Health-Related Risks From Uncontrolled Hypertension. Prev Chronic Dis 2018;15:170362. DOI: https://doi.org/10.5888/pcd15.170362.

\section{PEER REVIEWED}

\section{Abstract}

Uncontrolled hypertension, a common disorder, is associated with increased long-term risk of several serious conditions. Awareness of the health risks of uncontrolled hypertension is not well understood. We used data from a nationwide panel survey to assess the awareness of risk associated with uncontrolled hypertension, stratified by cardiovascular disease risk factors. Awareness of increased risk from uncontrolled hypertension was high for some outcomes (heart attack, heart failure, stroke), and low for others (kidney disease, dementia). Several disparities in awareness were found. Complementary clinical and public health interventions could be instituted to increase awareness and target people who are high risk.

\section{Objective}

Uncontrolled hypertension increases the risk of heart attack, heart failure, kidney disease, stroke, and cognitive decline (1-3). Low control rates are partly explained by lack of awareness of hypertension; improving awareness could lead to improved control rates $(4,5)$. However, few reports exist on what proportion of adults are aware of health risks from uncontrolled hypertension. We analyzed data from a national, web-based panel survey to assess awareness of risk of cardiovascular disease (CVD) and dementia associated with uncontrolled hypertension stratified by comorbidities, sociodemographic variables, and CVD risk factors.

\section{Methods}

HealthStyles is a web-based panel survey developed and licensed by Porter Novelli Public Services and conducted by GfK's KnowledgePanel (www.gfk.com/products-a-z/us/knowledgepanelunited-states/) as a probability-based online panel representative of the US adult population aged 18 or older. The survey was sent to 6,166 respondents in June and July of 2016; 4,203 completed the survey ( $68 \%$ response rate). The survey collected data on demographics, health conditions, health beliefs and awareness of health risks, and behaviors. Data were weighted for sex, age, household income, race/ethnicity, household size, education, census region, metropolitan status, and prior Internet access on the basis of the 2015 US Current Population Survey. All data were self-reported. Licensed data were provided; data did not include individual identifiers, was provided in aggregate form, and was granted an exemption by the Centers for Disease Control and Prevention institutional review board.

Primary variables were self-reported hypertension (classified as hypertensive or normotensive) and awareness of increased risk associated with uncontrolled hypertension for serious disorders (heart attack, heart failure, kidney disease, stroke, and dementia). Risk awareness was characterized by asking, "Which, if any, of the following are risks of uncontrolled blood pressure?" Answer choices were heart attack, heart failure, kidney disease, stroke, dementia, and none of these. Characteristics examined included: sex, age (18-44 y, 45-64 y, or $\geq 65 \mathrm{y}$ ), race/ethnicity (non-Hispanic white, non-Hispanic black, non-Hispanic other, or Hispanic), education (less than high school graduate, high school graduate, some college, or college graduate or more), annual household income $(<\$ 25,000, \$ 25,000-\$ 39,999, \$ 40,000-\$ 59,999$, or $\geq \$ 60,000)$, body mass index (BMI, $\left.\mathrm{kg} / \mathrm{m}^{2}\right)(<25.0=$ normal, $25.0-29.9=$ overweight, $\geq 30=$ obese), and CVD risk factors (current smoking, obesity), or comorbidities (atrial fibrillation, depression, heart failure, migraine, diabetes, stroke). 
We excluded 37 pregnant hypertensive respondents, and the final sample was 4,166 . We used Wald $\chi^{2}$ tests to assess differences in awareness among hypertensive adults by selected characteristics. Two-tailed $P$ values of $\leq .05$ were considered significant. All analyses were conducted using SAS version 9.3 (SAS Institute Inc).

\section{Results}

The prevalence of self-reported hypertension was $30.0 \%$ (Table 1), with significant differences by age, race/ethnicity, annual household income, education, BMI, and selected comorbidities. Hypertensive adults had significantly higher estimates of awareness for increased risk for stroke associated with uncontrolled hypertension compared with their normotensive counterparts $(90.9 \%$ vs $77.2 \%, P<.001)$. Similar patterns were seen among hypertensive adults versus normotensive adults for awareness of risk of heart attack $(88.4 \%$ vs $78.7 \%, P<.001)$, heart failure $(71.6 \%$ vs $65.8 \%, P=.002)$, and kidney disease $(38.5 \%$ vs $24.8 \%, P<.001)$. Though not significant, awareness for risk of dementia was markedly low in both groups: $8.7 \%$ for hypertensive adults and $7.9 \%$ for normotensive adults $(P=.47)$.

Among hypertensive adults, men were significantly more aware of increased risk for heart attack and heart failure associated with uncontrolled hypertension than women $(93.0 \%$ vs $83.9 \%, P<.001$, and $76.2 \%$ vs $67.0 \%, P .001$, respectively; Table 2). Compared with adults with lower annual household income, adults with higher household incomes had higher risk awareness estimates of for stroke, kidney disease and dementia. Non-Hispanic whites had higher risk awareness estimates than non-Hispanic blacks for heart attack $(90.9 \%$ vs $80.7 \%, P=.02)$, heart failure $(74.4 \%$ vs $62.8 \%, P$ $=.03)$, stroke $(93.7 \%$ vs $87.5 \%, P=.01)$, and dementia (9.7 vs 5.9, $P=.01)$.

Among hypertensive adults, respondents with depression were more likely to be aware of the risk of heart failure from uncontrolled hypertension than those without depression $(79.2 \%$ vs $69.8 \%, P=.01)$. Respondents with diabetes had no differences in awareness for all CVD outcomes compared with those without diabetes. Respondents with high cholesterol were more aware of the risks from uncontrolled hypertension for stroke than those without ( $94.1 \%$ vs $88.6 \%, P=.004)$. Respondents with 2 or more comorbidities had significantly higher risk awareness of heart attack and heart failure associated with uncontrolled hypertension than those with none or one of the conditions (Table 2).

\section{Discussion}

While awareness was high among most respondents for traditionally well-known CVD outcomes (heart attack, heart failure, stroke), it was remarkably lower for others (kidney disease, de- mentia), suggesting areas for focusing education. As previously reported $(6,7)$, we found notable socioeconomic and racial/ethnic differences in awareness of the risks of hypertension. For example, we found greater awareness of the association between uncontrolled hypertension and kidney disease, stroke, and dementia in high-income households than in low-income households. NonHispanic whites had greater risk awareness than non-Hispanic blacks, who have higher prevalence of hypertension and uncontrolled hypertension (8).

Education levels are associated with greater health literacy (7), which influences a patient's ability to understand hypertension education, complications from uncontrolled hypertension, and disparities in access to hypertension care (9). Increasing access to appropriate clinical care, supported by broad public health initiatives such as promoting use of community health workers to educate patients may help address the lack of awareness of risks from uncontrolled hypertension (7). Moreover, encouraging health care providers to more effectively communicate the dangers of uncontrolled hypertension can improve adherence to medications and prognosis (10). Additionally, risk awareness initiatives should tailor interventions to people with both diabetes and hypertension given the concurrent and heightened CVD and chronic kidney disease risks uncontrolled hypertension poses for them.

Initiatives — such as the National Institute of Neurological Disorders and Stroke's Mind Your Risks (https:// mindyourrisks.nih.gov/), World Kidney Day (www.worldkidneyday.org/), Million Hearts (https://millionhearts.hhs.gov/), Measure Up/Pressure Down (www.measureuppressuredown.com/), and Target BP (http://news.heart.org/aha-ama-launch-high-blood-pressure-initiative/) — should be expanded to reach more diverse populations to further increase awareness of the detrimental effects of uncontrolled hypertension (ie, impaired cognitive and kidney function).

Our study's limitation was that the survey was subject to recall bias, which is inherent in self-reported data and convenience sampling. The strength of our study is that the panel was drawn from a quota sampling to obtain a study group that matched the US population on 7 census demographics.

This study highlights the areas of need for educating populations who are unaware of the risks from uncontrolled hypertension. With nearly 35 million people with uncontrolled hypertension (4), effective interventions are needed to raise awareness of its risks. Public health initiatives should be replicated and expanded for more diverse populations to provide education on the risks of uncontrolled hypertension.

\footnotetext{
The opinions expressed by authors contributing to this journal do not necessarily reflect the opinions of the U.S. Department of Health and Human Services, the Public Health Service, the Centers for Disease Control and Prevention, or the authors' affiliated institutions.
} 


\section{Acknowledgments}

This is original work and no copyrighted material, instruments, or tools were used. HealthStyles is a copyrighted survey used with the permission of Porter Novelli. The findings and conclusions in this report are those of the authors and do not necessarily represent the official views of the Centers for Disease Control and Prevention. Authors have no conflicts of interest regarding funding or other issues related to this article and have no disclosures.

\section{Author Information}

Corresponding Author: Carma Ayala, PhD, MPH, RN, Division for Heart Disease and Stroke Prevention, National Center for Chronic Disease Prevention and Health Promotion, Centers for Disease Control and Prevention, 4770 Buford Highway NE, MS F73 Atlanta, GA 30341-3717 Telephone: 770-488-8072. Email: cia1@cdc.gov.

Author Affiliations: ${ }^{1}$ Centers for Disease Control and Prevention, National Center for Chronic Disease Prevention and Health Promotion, Division for Heart Disease and Stroke Prevention, Atlanta, Georgia. ${ }^{2}$ Emory University School of Medicine, Department of Internal Medicine, Atlanta, Georgia.

\section{References}

1. Control GBPU. Centers for Disease Control and Prevention; 2012. https://www.cdc.gov/vitalsigns/hypertension/. Accessed March 21, 2017.

2. Maesen B, Pison L, Meir M. Letter by Maesen et al regarding article, "Ablation of atrial fibrillation: patient selection, periprocedural anticoagulation, techniques, and preventive measures after ablation." Circulation 2017;135(1):e1-2.

3. National Institute of Neurological Disorders and Stroke (NINDS), National Institutes of Health. The dementias: hope through research. Washington (DC): National Institutes of Health; September 2013. https://catalog.ninds.nih.gov/ pubstatic//13-2252/13-2252.pdf Accessed March 21, 2017.

4. Hajjar I, Kotchen TA. Trends in prevalence, awareness, treatment, and control of hypertension in the United States, 1988-2000. JAMA 2003;290(2):199-206.

5. Yoon SS, Fryar CD, Carroll MD. Hypertension prevalence and control among adults: United States, 2011-2014. NCHS data brief, no 220. Hyattsville (MD): National Center for Health Statistics; 2015.

6. Gudmundsdottir H, Høieggen A, Stenehjem A, Waldum B, Os I. Hypertension in women: latest findings and clinical implications. Ther Adv Chronic Dis 2012;3(3):137-46.
7.Zimmerman E, Woolf S, Haley A. Population health: behavioral and social science insights: understanding the relationship between education and health. Us Department of Health and Human Services, Agency for Healthcare Research and Quality; September 2015. https://www.ahrq.gov/ professionals/education/curriculum-tools/population-health/ zimmerman.html Accessed March 23, 2017.

8. National Center for Health Statistics (NCHS). Health, United States, 2015: with special feature on racial and ethnic health disparities; 2016. https://www.cdc.gov/nchs/data/hus/ hus15.pdf\#054 Accessed April 12, 2017.

9. Dalton ARH, Vamos EP, Harris MJ, Netuveli G, Wachter RM, Majeed A, et al. Impact of universal health insurance coverage on hypertension management: a cross-national study in the United States and England. PLoS One 2014;9(1):e83705.

10. Swain S, Hariharan M, Rana S, Chivukula U, Thomas M. Doctor-patient communication: impact on adherence and prognosis among patients with primary hypertension. Psychol Stud (Mysore) 2015;60(1):25-32. https://link.springer.com/ article/10.1007/s12646-014-0291-5 Accessed October 28, 2016

The opinions expressed by authors contributing to this journal do not necessarily reflect the opinions of the U.S. Department of Health and Human Services, the Public Health Service, the Centers for Disease Control and Prevention, or the authors' affiliated institutions. 


\section{Tables}

Table 1. Weighted Percentage ${ }^{a}$ by Sociodemographic Characteristics, Cardiovascular Disease Risk Factors (CVD), and Awareness of Increased CVD Risks Associated With Uncontrolled Blood Pressure Among Respondents $(\mathrm{N}=4,166)$, HealthStyles, $2016^{\mathrm{b}}$

\begin{tabular}{|c|c|c|c|c|}
\hline \multirow[b]{3}{*}{ Variable } & \multirow[b]{3}{*}{ Total, N (\%) } & \multicolumn{3}{|c|}{ Self-Reported Hypertension } \\
\hline & & Yes & No & \multirow[b]{2}{*}{$P$ Value $^{\mathrm{C}}$} \\
\hline & & \multicolumn{2}{|c|}{ N (\%) $[95 \% \mathrm{Cl}]$} & \\
\hline Total $^{d}$ & $4,166(100)$ & $1,414(30.0)[28.3-31.6]$ & 2,752 (70.0) [68.4-71.7] & NA \\
\hline \multicolumn{5}{|c|}{ Demographic Characteristic } \\
\hline \multicolumn{5}{|l|}{ Age, $y$} \\
\hline $18-44$ & $1,457(46.1)$ & 188 (17.3) [14.8-19.9] & 1,269 (58.4) [56.2-60.5] & \multirow[t]{3}{*}{$<.001$} \\
\hline $45-64$ & $954(19.3)$ & $567(37.6)[34.7-40.6]$ & 387 (11.4) [10.2-12.7] & \\
\hline$\geq 65$ & $1,755(34.7)$ & 659 (45.1) [42.0-48.1] & $1,096(30.2)[28.3-32.1]$ & \\
\hline \multicolumn{5}{|l|}{ Sex } \\
\hline Male & $1,996(48.7)$ & $727(49.7)$ [46.6-52.8] & $1,269(48.2)[46.0-50.5]$ & \multirow[t]{2}{*}{.45} \\
\hline Female & $2,170(51.3)$ & $687(50.3)[47.2-53.4]$ & $1,483(51.8)$ [49.5-54.0] & \\
\hline \multicolumn{5}{|l|}{ Race/ethnicity } \\
\hline Non-Hispanic white & $3,078(65.2)$ & $1,037(64.0)[60.8-67.2]$ & $2,041(65.7)[63.4-68.1]$ & \multirow[t]{4}{*}{$<.001$} \\
\hline Non-Hispanic black & $421(11.7)$ & 189 (17.3) [14.7-19.8] & $232(9.3)[7.9-10.7]$ & \\
\hline Hispanic & $466(15.4)$ & 139 (13.5) [11.1-15.9] & $327(16.2)[14.4-18.1]$ & \\
\hline Non-Hispanic other ${ }^{\mathrm{e}}$ & $201(7.7)$ & 49 (5.2) [3.5-7.0] & $152(8.7)[7.1-10.3]$ & \\
\hline \multicolumn{5}{|l|}{ Education } \\
\hline$<$ High school graduate & $275(12.1)$ & $114(14.7)[12.0-17.4]$ & 161 (11.0) [9.2-12.7] & \multirow{4}{*}{$<.001$} \\
\hline High school graduate & $1,238(29.8)$ & 498 (34.7) [31.8-37.6] & 740 (27.6) [25.6-29.7] & \\
\hline Some college & $1,260(28.4)$ & $418(26.7)$ [24.1-29.3] & $842(29.1)[27.1-31.1]$ & \\
\hline College graduate or more & 1,393 (29.8) & 384 (23.9) [21.3-26.4] & 1,009 (32.3) [30.3-34.4] & \\
\hline \multicolumn{5}{|l|}{ Annual household income, $\$$} \\
\hline$<25,000$ & 709 (17.4) & $303(22.4)[19.7-25.1]$ & 406 (15.2) [13.5-16.9] & \multirow{4}{*}{$<.001$} \\
\hline $25,000-39,999$ & $670(13.1)$ & 256 (15.1) [13.1-17.2] & 414 (12.2) [10.8-13.6] & \\
\hline $40,000-59,999$ & $683(15.9)$ & 236 (16.1) [13.9-18.3] & $447(15.8)[14.1-17.4]$ & \\
\hline$\geq 60,000$ & $2,104(53.7)$ & $619(46.4)[43.3-49.4]$ & $1,485(56.8)[54.5-59.0]$ & \\
\hline \multicolumn{5}{|l|}{$\mathrm{BMI}, \mathrm{kg} / \mathrm{m}^{2}(\mathrm{~N}=4,080)$} \\
\hline$<25.0$ (normal) & 1,335 (35.8) & 286 (22.1) [19.4-24.7] & 1,049 (41.6) [39.4-43.9] & \multirow{2}{*}{$<.001$} \\
\hline 25.0-29.9 (overweight) & $1,377(33.0)$ & 453 (32.4) [29.5-35.3] & $924(33.2)[31.1-35.4]$ & \\
\hline
\end{tabular}

Abbreviation: BMI, body mass index.

a Data were weighted for sex, age, annual household income, race/ethnicity, household size, education, census region, metropolitan status, and prior Internet access based on 2015 US Current Population Survey. Of the 4,203 respondents, 37 pregnant women with hypertension were excluded.

${ }^{\mathrm{b}}$ HealthStyles is a web-based panel survey developed and licensed by Porter Novelli Public Services and conducted by GfK's KnowledgePanel.

${ }^{c} P$ values calculated by using Wald $x^{2}$ test.

${ }^{\mathrm{d}}$ Total number of respondents with self-reported hypertension who answered the question.

${ }^{\mathrm{e}}$ The overall non-Hispanic other group sample consisted of 24 American Indian/Alaska Natives, 111 Asians, and 101 people of other races. The hypertensive nonHispanic other group sample consisted of 3 American Indian/Alaska Natives, 25 Asians, and 24 people of other races, sample sizes that were too small to use as individual groups for analyses.

(continued on next page)

The opinions expressed by authors contributing to this journal do not necessarily reflect the opinions of the U.S. Department of Health and Human Services, the Public Health Service, the Centers for Disease Control and Prevention, or the authors' affiliated institutions. 
(continued)

Table 1. Weighted Percentage ${ }^{a}$ by Sociodemographic Characteristics, Cardiovascular Disease Risk Factors (CVD), and Awareness of Increased CVD Risks Associated With Uncontrolled Blood Pressure Among Respondents $(\mathrm{N}=4,166)$, HealthStyles, $2016^{\mathrm{b}}$

\begin{tabular}{|c|c|c|c|c|}
\hline \multirow[b]{3}{*}{ Variable } & \multirow[b]{3}{*}{ Total, N (\%) } & \multicolumn{3}{|c|}{ Self-Reported Hypertension } \\
\hline & & Yes & No & \multirow[b]{2}{*}{ P Value ${ }^{c}$} \\
\hline & & \multicolumn{2}{|c|}{$\mathrm{N}(\%)[95 \% \mathrm{Cl}]$} & \\
\hline$\geq 30.0$ (obese) & $1,368(31.2)$ & 638 (45.5) [42.4-48.6] & $730(25.2)[23.2-27.1]$ & \\
\hline \multicolumn{5}{|c|}{ Comorbidities } \\
\hline \multicolumn{5}{|c|}{ Current smoker $(\mathrm{N}=4,151)$} \\
\hline Yes & $507(12.4)$ & 165 (13.0) [10.8-15.2] & 342 (12.1) [10.6-13.5] & \multirow{2}{*}{.51} \\
\hline No & $3,644(87.6)$ & $1,245(87.0)[84.8-89.2]$ & 2,399 (87.9) [86.5-89.4] & \\
\hline \multicolumn{5}{|c|}{ Diabetes $(\mathrm{N}=4,133)$} \\
\hline Yes & $446(10.0)$ & $314(22.7)[20.1-25.3]$ & $132(4.6)[3.6-5.5]$ & \multirow{2}{*}{$<.001$} \\
\hline No & $3,687(90.0)$ & 1,095 (77.3) [74.7-79.9] & $2,592(95.4)[94.5-96.4]$ & \\
\hline \multicolumn{5}{|c|}{ High cholesterol $(\mathrm{N}=4,133)$} \\
\hline Yes & $897(18.9)$ & $617(42.0)[38.9-45.0]$ & $280(9.0)[7.8-10.3]$ & \multirow{2}{*}{$<.001$} \\
\hline No & $3,236(81.1)$ & $792(58.0)[55.0-61.1]$ & 2,444 (91.0) [89.7-92.2] & \\
\hline \multicolumn{5}{|c|}{ Congestive heart failure $(N=4,133)$} \\
\hline Yes & $43(1.0)$ & $32(2.6)[1.5-3.7]$ & $11(0.3)[0.1-0.5]$ & \multirow{2}{*}{$<.001$} \\
\hline No & $4,090(99.0)$ & $1,377(97.4)[96.3-98.5]$ & 2,713 (99.7) [99.5-99.9] & \\
\hline \multicolumn{5}{|c|}{ Any cancer (except skin) $(\mathrm{N}=4,133)$} \\
\hline Yes & $68(1.6)$ & 39 (2.8) [1.8-3.9] & 29 (1.0) [0.6-1.4] & \multirow{2}{*}{.001} \\
\hline No & $4,065(98.4)$ & $1,370(97.2)[96.1-98.2]$ & 2,695 (99.0) [98.6-99.4] & \\
\hline \multicolumn{5}{|c|}{ Migraines $(\mathrm{N}=4,133)$} \\
\hline Yes & $342(8.2)$ & $98(7.4)[5.7-9.2]$ & $244(8.5)[7.3-9.8]$ & \multirow[t]{2}{*}{.31} \\
\hline No & $3,791(91.8)$ & 1,311 (92.6) [90.8-94.3] & 2,480 (91.5) [90.2-92.7] & \\
\hline \multicolumn{5}{|c|}{ Atrial fibrillation $(\mathrm{N}=4,133)$} \\
\hline Yes & $77(1.5)$ & 55 (3.3) [2.4-4.3] & $22(0.8)[0.4-1.1]$ & \multirow{2}{*}{$<.001$} \\
\hline No & $4,056(98.5)$ & 1,354 (96.7) [95.7-97.6] & 2,702 (99.2) [98.9-99.6] & \\
\hline \multicolumn{5}{|c|}{ Depression $(\mathrm{N}=4,133)$} \\
\hline Yes & $577(13.8)$ & 252 (18.3) [15.9-20.8] & 325 (11.8) [10.3-13.3] & \multirow{2}{*}{$<.001$} \\
\hline No & $3,556(86.2)$ & $1,157(81.7)[79.2-84.1]$ & 2,399 (88.2) [86.7-89.7] & \\
\hline \multicolumn{5}{|c|}{ Multiple comorbidities } \\
\hline None & $2,090(53.9)$ & $431(31.9)[29.0-34.8]$ & 1,659 (63.3) [61.1-65.4] & \multirow{2}{*}{$<.001$} \\
\hline 1 & $1,347(30.3)$ & 557 (37.4) [34.4-40.3] & 790 (27.3) [25.3-29.3] & \\
\hline
\end{tabular}

Abbreviation: BMI, body mass index.

a Data were weighted for sex, age, annual household income, race/ethnicity, household size, education, census region, metropolitan status, and prior Internet access based on 2015 US Current Population Survey. Of the 4,203 respondents, 37 pregnant women with hypertension were excluded.

${ }^{\mathrm{b}}$ HealthStyles is a web-based panel survey developed and licensed by Porter Novelli Public Services and conducted by GfK's KnowledgePanel.

${ }^{c} P$ values calculated by using Wald $x^{2}$ test.

${ }^{\mathrm{d}}$ Total number of respondents with self-reported hypertension who answered the question.

${ }^{\mathrm{e}}$ The overall non-Hispanic other group sample consisted of 24 American Indian/Alaska Natives, 111 Asians, and 101 people of other races. The hypertensive nonHispanic other group sample consisted of 3 American Indian/Alaska Natives, 25 Asians, and 24 people of other races, sample sizes that were too small to use as individual groups for analyses.

(continued on next page)

The opinions expressed by authors contributing to this journal do not necessarily reflect the opinions of the U.S. Department of Health and Human Services, the Public Health Service, the Centers for Disease Control and Prevention, or the authors' affiliated institutions. 
(continued)

Table 1. Weighted Percentage ${ }^{a}$ by Sociodemographic Characteristics, Cardiovascular Disease Risk Factors (CVD), and Awareness of Increased CVD Risks Associated With Uncontrolled Blood Pressure Among Respondents $(\mathrm{N}=4,166)$, HealthStyles, $2016^{\mathrm{b}}$

\begin{tabular}{|c|c|c|c|c|}
\hline \multirow[b]{3}{*}{ Variable } & \multirow[b]{3}{*}{ Total, $\mathrm{N}(\%)$} & \multicolumn{3}{|c|}{ Self-Reported Hypertension } \\
\hline & & Yes & No & \multirow[b]{2}{*}{$P$ Value $^{c}$} \\
\hline & & \multicolumn{2}{|c|}{$\mathrm{N}(\%)[95 \% \mathrm{Cl}]$} & \\
\hline$\geq 2$ & $681(15.8)$ & $417(30.7)[27.8-33.6]$ & $264(9.4)[8.1-10.7]$ & \\
\hline \multicolumn{5}{|c|}{ Awareness of conditions associated with uncontrolled hypertension $(N=4,129)$} \\
\hline Heart attack & $3,461(81.7)$ & $1,250(88.4)[86.4-90.4]$ & $2,211(78.7)[76.8-80.7]$ & $<.001$ \\
\hline Heart failure & $2,851(67.6)$ & $1,021(71.6)[68.8-74.4]$ & $1,830(65.8)[63.7-68.0]$ & .002 \\
\hline Kidney disease & $1,208(28.9)$ & $543(38.5)[35.5-41.6]$ & $665(24.8)[22.8-26.8]$ & $<.001$ \\
\hline Stroke & $3,481(81.3)$ & 1,299 (90.9) [89.0-92.8] & $2,182(77.2)[75.2-79.2]$ & $<.001$ \\
\hline Dementia & $336(8.2)$ & $123(8.7)[7.0-10.5]$ & 213 (7.9) [6.6-9.2] & .47 \\
\hline
\end{tabular}

Abbreviation: BMI, body mass index.

a Data were weighted for sex, age, annual household income, race/ethnicity, household size, education, census region, metropolitan status, and prior Internet access based on 2015 US Current Population Survey. Of the 4,203 respondents, 37 pregnant women with hypertension were excluded.

${ }^{\mathrm{b}}$ HealthStyles is a web-based panel survey developed and licensed by Porter Novelli Public Services and conducted by GfK's KnowledgePanel.

${ }^{c} P$ values calculated by using Wald $x^{2}$ test.

${ }^{\mathrm{d}}$ Total number of respondents with self-reported hypertension who answered the question.

${ }^{\mathrm{e}}$ The overall non-Hispanic other group sample consisted of 24 American Indian/Alaska Natives, 111 Asians, and 101 people of other races. The hypertensive nonHispanic other group sample consisted of 3 American Indian/Alaska Natives, 25 Asians, and 24 people of other races, sample sizes that were too small to use as individual groups for analyses.

The opinions expressed by authors contributing to this journal do not necessarily reflect the opinions of the U.S. Department of Health and Human Services, the Public Health Service, the Centers for Disease Control and Prevention, or the authors' affiliated institutions. 
Table 2. Weighted Percentage ${ }^{a}$ of Awareness of Health Risks Associated with Uncontrolled Hypertension Among Respondents Who Self-Reported Hypertension (N = 1,408), by Selected Characteristics, HealthStyles ${ }^{b}, 2016$

\begin{tabular}{|c|c|c|c|c|c|c|c|c|c|c|}
\hline \multirow[b]{2}{*}{ Characteristic } & \multicolumn{2}{|c|}{ Heart Attack } & \multicolumn{2}{|c|}{ Heart Failure } & \multicolumn{2}{|c|}{ Kidney Disease } & \multicolumn{2}{|c|}{ Stroke } & \multicolumn{2}{|c|}{ Dementia } \\
\hline & $\%(95 \% \mathrm{Cl})$ & $P$ Value & $\%(95 \% \mathrm{Cl})$ & $P$ Value & $\%(95 \% \mathrm{Cl})$ & $P$ Value & $\%(95 \% \mathrm{Cl})$ & $P$ Value & $\%(95 \% \mathrm{Cl})$ & PValue ${ }^{c}$ \\
\hline Total & $\begin{array}{l}88.4 \\
(86.4-90.4)\end{array}$ & NA & $\begin{array}{l}71.6 \\
(68.8-74.4)\end{array}$ & NA & $\begin{array}{l}38.5 \\
(35.5-41.6)\end{array}$ & NA & $\begin{array}{l}90.9 \\
(89.0-92.8)\end{array}$ & NA & $\begin{array}{l}8.7 \\
(7.0-10.5)\end{array}$ & NA \\
\hline \multicolumn{11}{|l|}{ Demographic Characteristic } \\
\hline \multicolumn{11}{|l|}{ Age, y } \\
\hline $18-44$ & \begin{tabular}{|l}
90.4 \\
$(85.5-95.3)$
\end{tabular} & \multirow{3}{*}{.61} & $\begin{array}{l}73.6 \\
(66.2-81.0) \\
\end{array}$ & \multirow{3}{*}{.52} & \begin{tabular}{|l|}
34.3 \\
$(26.4-42.2)$ \\
\end{tabular} & \multirow{3}{*}{.40} & \begin{tabular}{|l|}
84.2 \\
$(77.6-90.8)$ \\
\end{tabular} & \multirow{3}{*}{.05} & $5.2(1.7-8.7)$ & \multirow{3}{*}{.12} \\
\hline $45-64$ & $\begin{array}{l}88.6 \\
(85.4-91.8)\end{array}$ & & $\begin{array}{l}69.5 \\
(64.8-74.1) \\
\end{array}$ & & $\begin{array}{l}40.6 \\
(35.8-45.5)\end{array}$ & & $\begin{array}{l}93.1 \\
(90.6-95.5) \\
\end{array}$ & & $\begin{array}{l}9.2 \\
(6.1-12.2) \\
\end{array}$ & \\
\hline$\geq 65$ & $\begin{array}{l}87.5 \\
(84.6-90.5)\end{array}$ & & \begin{tabular}{|l}
72.6 \\
$(68.6-76.6)$
\end{tabular} & & \begin{tabular}{|l}
38.4 \\
$(34.1-42.8)$
\end{tabular} & & $\begin{array}{l}91.7 \\
(89.0-94.4)\end{array}$ & & $\begin{array}{l}9.7 \\
(7.1-12.3)\end{array}$ & \\
\hline \multicolumn{11}{|l|}{ Sex } \\
\hline Male & $\begin{array}{l}93.0 \\
(91.0-95.0)\end{array}$ & \multirow{2}{*}{$<.001$} & $\begin{array}{l}76.2 \\
(72.5-79.9)\end{array}$ & \multirow{2}{*}{.001} & $\begin{array}{l}39.5 \\
(35.3-43.7)\end{array}$ & & $\begin{array}{l}91.2 \\
(88.4-93.9)\end{array}$ & \multirow{2}{*}{.79} & $\begin{array}{l}7.8 \\
(5.6-10.1)\end{array}$ & \multirow{2}{*}{.33} \\
\hline Female & $\begin{array}{l}83.9 \\
(80.5-87.3)\end{array}$ & & $\begin{array}{l}67.0 \\
(62.8-71.3)\end{array}$ & & \begin{tabular}{|l}
37.6 \\
$(33.3-42.0)$
\end{tabular} & .55 & $\begin{array}{l}90.7 \\
(88.0-93.3)\end{array}$ & & $\begin{array}{l}9.6 \\
(6.9-12.3)\end{array}$ & \\
\hline \multicolumn{11}{|l|}{ Race/ethnicity } \\
\hline Non-Hispanic white & $\begin{array}{l}90.9 \\
(89.0-92.9)\end{array}$ & \multirow{4}{*}{.02} & $\begin{array}{l}74.4 \\
(71.3-77.5)\end{array}$ & \multirow{4}{*}{.03} & $\begin{array}{l}37.1 \\
(33.8-40.4)\end{array}$ & \multirow{4}{*}{.69} & $\begin{array}{l}93.7 \\
(91.9-95.4)\end{array}$ & \multirow{4}{*}{.01} & $\begin{array}{l}9.7 \\
(7.6-11.8)\end{array}$ & \multirow{4}{*}{.01} \\
\hline Non-Hispanic black & \begin{tabular}{|l}
80.7 \\
$(74.2-87.2)$ \\
\end{tabular} & & $\begin{array}{l}62.8 \\
(54.8-70.8) \\
\end{array}$ & & \begin{tabular}{|l}
40.7 \\
$(32.4-49.0)$ \\
\end{tabular} & & \begin{tabular}{|l}
87.5 \\
$(81.3-93.7)$ \\
\end{tabular} & & $5.9(2.1-9.8)$ & \\
\hline Hispanic & $\begin{array}{l}87.5 \\
(80.9-94.1) \\
\end{array}$ & & $\begin{array}{l}66.9 \\
(57.8-75.9) \\
\end{array}$ & & $\begin{array}{l}40.3 \\
(30.7-50.0)\end{array}$ & & \begin{tabular}{|l}
86.3 \\
$(79.4-93.2)$ \\
\end{tabular} & & $4.0(0.9-7.0)$ & \\
\hline Non-Hispanic other ${ }^{d}$ & $\begin{array}{l}85.5 \\
(74.2-96.8)\end{array}$ & & \begin{tabular}{|l}
77.7 \\
$(63.3-92.1)$
\end{tabular} & & \begin{tabular}{|l}
44.8 \\
$(27.4-62.2)$ \\
\end{tabular} & & \begin{tabular}{|l}
80.7 \\
$(68.3-93.2)$ \\
\end{tabular} & & $\begin{array}{l}18.0 \\
(3.1-32.8)\end{array}$ & \\
\hline \multicolumn{11}{|l|}{ Education } \\
\hline$<$ High school graduate & $\begin{array}{l}88.1 \\
(81.6-94.7)\end{array}$ & \multirow{4}{*}{.60} & $\begin{array}{l}61.8 \\
(51.6-71.9) \\
\end{array}$ & \multirow{4}{*}{.06} & $\begin{array}{l}36.9 \\
(26.7-47.2) \\
\end{array}$ & \multirow{4}{*}{.001} & \begin{tabular}{|l}
84.9 \\
$(77.2-92.5)$ \\
\end{tabular} & \multirow{4}{*}{.23} & $3.7(0.2-7.2)$ & \multirow{4}{*}{.005} \\
\hline High school graduate & $\begin{array}{l}87.2 \\
(83.8-90.7) \\
\end{array}$ & & \begin{tabular}{|l}
70.3 \\
$(65.7-74.9)$ \\
\end{tabular} & & \begin{tabular}{|l}
34.0 \\
$(29.3-38.7)$ \\
\end{tabular} & & \begin{tabular}{|l|}
90.7 \\
$(87.6-93.9)$ \\
\end{tabular} & & \begin{tabular}{|l|}
7.6 \\
$(4.9-10.3)$ \\
\end{tabular} & \\
\hline Some college & $\begin{array}{l}88.3 \\
(84.7-91.9)\end{array}$ & & $\begin{array}{l}74.9 \\
(70.0-79.8) \\
\end{array}$ & & \begin{tabular}{|l}
35.2 \\
$(30.0-40.4)$ \\
\end{tabular} & & \begin{tabular}{|l}
92.7 \\
$(89.6-95.8)$ \\
\end{tabular} & & $\begin{array}{l}7.4 \\
(4.7-10.1)\end{array}$ & \\
\hline College graduate or more & $\begin{array}{l}90.5 \\
(87.1-94.0)\end{array}$ & & $\begin{array}{l}75.7 \\
(70.8-80.6)\end{array}$ & & $\begin{array}{l}49.8 \\
(43.8-55.8)\end{array}$ & & $\begin{array}{l}92.9 \\
(89.9-95.9)\end{array}$ & & $\begin{array}{l}14.8 \\
(10.0-19.6)\end{array}$ & \\
\hline
\end{tabular}

Annual household income, \$

Abbreviations: $\mathrm{Cl}$, confidence interval; NA, not applicable.

a Data were weighted for sex, age, annual household income, race/ethnicity, household size, education, census region, metropolitan status, and prior Internet access based on 2015 US Current Population Survey. All data were self-reported. Of 4,203 respondents, only 1,408 reported being aware of the risks associated with hypertension.

${ }^{\mathrm{b}}$ HealthStyles is a web-based panel survey developed and licensed by Porter Novelli Public Services and conducted by GfK's KnowledgePanel.

${ }^{c} P$ values calculated by using Wald $x^{2}$ test.

${ }^{d}$ The overall non-Hispanic other group sample consisted of 24 American Indian/Alaska Natives, 111 Asians, and 101 peopole of other races. The hypertensive non-Hispanic other group sample consisted of 3 American Indian/Alaska Natives, 25 Asians, and 24 people of other races; these sample sizes were too small to use as individual groups for analyses.

e Sample size was too small for reliable results.

${ }^{f}$ Comorbidities were heart attack, heart failure, kidney disease, stroke, and dementia.

(continued on next page)

The opinions expressed by authors contributing to this journal do not necessarily reflect the opinions of the U.S. Department of Health and Human Services, the Public Health Service, the Centers for Disease Control and Prevention, or the authors' affiliated institutions. 
(continued)

Table 2. Weighted Percentage ${ }^{a}$ of Awareness of Health Risks Associated with Uncontrolled Hypertension Among Respondents Who Self-Reported Hypertension (N = 1,408), by Selected Characteristics, HealthStyles ${ }^{b}, 2016$

\begin{tabular}{|c|c|c|c|c|c|c|c|c|c|c|}
\hline \multirow[b]{2}{*}{ Characteristic } & \multicolumn{2}{|c|}{ Heart Attack } & \multicolumn{2}{|c|}{ Heart Failure } & \multicolumn{2}{|c|}{ Kidney Disease } & \multicolumn{2}{|c|}{ Stroke } & \multicolumn{2}{|c|}{ Dementia } \\
\hline & $\%(95 \% \mathrm{Cl})$ & $P$ Value & $\%(95 \% \mathrm{Cl})$ & $P$ Value & $\%(95 \% \mathrm{Cl})$ & $P$ Value & $\%(95 \% \mathrm{Cl})$ & $P$ Value & $\%(95 \% \mathrm{Cl})$ & $P$ Value $^{c}$ \\
\hline$<25,000$ & $\begin{array}{l}85.1 \\
(79.8-90.4)\end{array}$ & \multirow{4}{*}{.07} & $\begin{array}{l}67.0 \\
(60.1-73.8)\end{array}$ & \multirow{4}{*}{.19} & $\begin{array}{l}27.8 \\
(21.8-33.9)\end{array}$ & \multirow{4}{*}{.001} & $\begin{array}{l}84.8 \\
(79.4-90.2)\end{array}$ & \multirow{4}{*}{.03} & $4.9(2.1-7.8)$ & \multirow{4}{*}{.04} \\
\hline $25,000-39,999$ & $\begin{array}{l}89.5 \\
(85.6-93.3) \\
\end{array}$ & & $\begin{array}{l}69.1 \\
(62.1-76.1) \\
\end{array}$ & & $\begin{array}{l}37.5 \\
(30.0-44.9) \\
\end{array}$ & & $\begin{array}{l}92.4 \\
(88.6-96.1) \\
\end{array}$ & & $\begin{array}{l}7.1 \\
(3.6-10.6)\end{array}$ & \\
\hline $40,000-59,999$ & $\begin{array}{l}84.5 \\
(78.9-90.1)\end{array}$ & & \begin{tabular}{|l}
71.2 \\
$(64.5-77.9)$
\end{tabular} & & $\begin{array}{l}40.8 \\
(33.4-48.2)\end{array}$ & & $\begin{array}{l}90.4 \\
(85.2-95.7)\end{array}$ & & $\begin{array}{l}10.2 \\
(5.7-14.7)\end{array}$ & \\
\hline$\geq 60,000$ & $\begin{array}{l}91.1 \\
(88.6-93.6)\end{array}$ & & \begin{tabular}{|l}
67.0 \\
$(60.1-73.8)$
\end{tabular} & & $\begin{array}{l}43.2 \\
(38.7-47.8)\end{array}$ & & \begin{tabular}{|l}
93.5 \\
$(91.2-95.9)$
\end{tabular} & & $\begin{array}{l}10.5 \\
(7.6-13.5)\end{array}$ & \\
\hline \multicolumn{11}{|l|}{$\mathrm{BMI}, \mathrm{kg} / \mathrm{m}^{2}$} \\
\hline$<25.0$ (normal) & $\begin{array}{l}83.4 \\
(77.9-88.9)\end{array}$ & \multirow{3}{*}{.09} & \begin{tabular}{|l}
68.7 \\
$(62.2-75.1)$ \\
\end{tabular} & & $\begin{array}{l}36.0 \\
(29.5-42.4)\end{array}$ & \multirow{3}{*}{.34} & $\begin{array}{l}88.9 \\
(84.3-93.5)\end{array}$ & & $\begin{array}{l}9.3 \\
(5.7-12.8)\end{array}$ & \multirow{3}{*}{.79} \\
\hline 25.0-29.9 (overweight) & $\begin{array}{l}89.0 \\
(85.7-92.3) \\
\end{array}$ & & \begin{tabular}{|l}
71.1 \\
$(66.0-76.3)$ \\
\end{tabular} & & $\begin{array}{l}36.1 \\
(30.8-41.4)\end{array}$ & & \begin{tabular}{|l|}
91.7 \\
$(88.5-94.9)$
\end{tabular} & & $\begin{array}{l}8.9 \\
(5.5-12.4)\end{array}$ & \\
\hline$\geq 30.0$ (obese) & $\begin{array}{l}90.4 \\
(87.8-93.0)\end{array}$ & & $\begin{array}{l}72.9 \\
(68.9-76.9)\end{array}$ & .55 & $\begin{array}{l}40.6 \\
(36.1-45.2)\end{array}$ & & $\begin{array}{l}91.2 \\
(88.3-94.1)\end{array}$ & .60 & $\begin{array}{l}7.9 \\
(5.4-10.4)\end{array}$ & \\
\hline \multicolumn{11}{|l|}{ Comorbidities } \\
\hline \multicolumn{11}{|l|}{ Current smoker } \\
\hline Yes & $\begin{array}{l}89.9 \\
(84.2-95.7)\end{array}$ & \multirow{2}{*}{.57} & $\begin{array}{l}72.8 \\
(64.1-81.4) \\
\end{array}$ & \multirow{2}{*}{.76} & $\begin{array}{l}27.7 \\
(19.8-35.7)\end{array}$ & \multirow{2}{*}{.007} & $\begin{array}{l}91.0 \\
(85.1-97.0)\end{array}$ & \multirow{2}{*}{$>.99$} & $\begin{array}{l}10.1 \\
(5.3-14.9)\end{array}$ & \multirow{2}{*}{.55} \\
\hline No & $\begin{array}{l}88.1 \\
(86.0-90.3)\end{array}$ & & \begin{tabular}{|l}
71.3 \\
$(68.4-74.3)$
\end{tabular} & & $\begin{array}{l}40.0 \\
(36.8-43.2)\end{array}$ & & $\begin{array}{l}91.0 \\
(88.9-93.0)\end{array}$ & & $\begin{array}{l}8.6 \\
(6.7-10.5)\end{array}$ & \\
\hline \multicolumn{11}{|l|}{ Diabetes } \\
\hline Yes & $\begin{array}{l}88.4 \\
(84.1-92.7)\end{array}$ & \multirow{2}{*}{.98} & \begin{tabular}{|l}
72.7 \\
$(66.7-78.7)$
\end{tabular} & \multirow{2}{*}{.65} & $\begin{array}{l}42.9 \\
(36.2-49.5)\end{array}$ & \multirow{2}{*}{.15} & $\begin{array}{l}90.4 \\
(86.1-94.7)\end{array}$ & \multirow{2}{*}{.79} & $\begin{array}{l}9.1 \\
(5.1-13.1)\end{array}$ & \multirow{2}{*}{.87} \\
\hline No & $\begin{array}{l}88.4 \\
(86.1-90.6)\end{array}$ & & \begin{tabular}{|l}
71.2 \\
$(68.0-74.4)$
\end{tabular} & & $\begin{array}{l}37.3 \\
(33.9-40.7)\end{array}$ & & $\begin{array}{l}91.0 \\
(88.9-93.2)\end{array}$ & & $\begin{array}{l}8.7 \\
(6.7-10.6)\end{array}$ & \\
\hline \multicolumn{11}{|l|}{ High cholesterol } \\
\hline Yes & $\begin{array}{l}89.9 \\
(87.0-92.8) \\
\end{array}$ & \multirow{2}{*}{.20} & \begin{tabular}{|l}
73.7 \\
$(69.6-77.8)$ \\
\end{tabular} & \multirow{2}{*}{.20} & \begin{tabular}{|l}
40.3 \\
$(35.7-44.9)$ \\
\end{tabular} & \multirow{2}{*}{.34} & \begin{tabular}{|l}
94.1 \\
$(91.8-96.3)$ \\
\end{tabular} & \multirow{2}{*}{-.004} & $\begin{array}{l}8.8 \\
(6.0-11.7) \\
\end{array}$ & 95 \\
\hline No & $\begin{array}{l}87.3 \\
(84.5-90.0)\end{array}$ & & $\begin{array}{l}70.0 \\
(66.1-73.8)\end{array}$ & & \begin{tabular}{|l}
37.3 \\
$(33.3-41.3)$
\end{tabular} & & $\begin{array}{l}88.6 \\
(85.7-91.5)\end{array}$ & & $\begin{array}{l}8.7 \\
(6.5-11.0)\end{array}$ & ת \\
\hline Congestive heart failure & & & & & & & & & & \\
\hline Yes & 100.0 & $N A^{e}$ & $\begin{array}{l}93.7 \\
(86.9-100) \\
\end{array}$ & $N A^{e}$ & \begin{tabular}{|l}
65.7 \\
$(46.5-84.8)$ \\
\end{tabular} & .02 & \begin{tabular}{|l}
98.2 \\
$(94.6-100.0)$ \\
\end{tabular} & $N A^{c}$ & \begin{tabular}{|l}
29.5 \\
$(8.8-50.3)$ \\
\end{tabular} & .08 \\
\hline
\end{tabular}

Abbreviations: $\mathrm{Cl}$, confidence interval; NA, not applicable.

a Data were weighted for sex, age, annual household income, race/ethnicity, household size, education, census region, metropolitan status, and prior Internet access based on 2015 US Current Population Survey. All data were self-reported. Of 4,203 respondents, only 1,408 reported being aware of the risks associated with hypertension.

${ }^{\mathrm{b}}$ HealthStyles is a web-based panel survey developed and licensed by Porter Novelli Public Services and conducted by GfK's KnowledgePanel.

${ }^{c} P$ values calculated by using Wald $x^{2}$ test.

${ }^{d}$ The overall non-Hispanic other group sample consisted of 24 American Indian/Alaska Natives, 111 Asians, and 101 peopole of other races. The hypertensive non-Hispanic other group sample consisted of 3 American Indian/Alaska Natives, 25 Asians, and 24 people of other races; these sample sizes were too small to use as individual groups for analyses.

${ }^{\text {e }}$ Sample size was too small for reliable results.

${ }^{f}$ Comorbidities were heart attack, heart failure, kidney disease, stroke, and dementia.

(continued on next page)

The opinions expressed by authors contributing to this journal do not necessarily reflect the opinions of the U.S. Department of Health and Human Services, the Public Health Service, the Centers for Disease Control and Prevention, or the authors' affiliated institutions. 
(continued)

Table 2. Weighted Percentage ${ }^{a}$ of Awareness of Health Risks Associated with Uncontrolled Hypertension Among Respondents Who Self-Reported Hypertension (N = 1,408), by Selected Characteristics, HealthStyles ${ }^{b}, 2016$

\begin{tabular}{|c|c|c|c|c|c|c|c|c|c|c|}
\hline \multirow[b]{2}{*}{ Characteristic } & \multicolumn{2}{|c|}{ Heart Attack } & \multicolumn{2}{|c|}{ Heart Failure } & \multicolumn{2}{|c|}{ Kidney Disease } & \multicolumn{2}{|c|}{ Stroke } & \multicolumn{2}{|c|}{ Dementia } \\
\hline & $\%(95 \% \mathrm{Cl})$ & $P$ Value & $\%(95 \% \mathrm{Cl})$ & $P$ Value & $\%(95 \% \mathrm{Cl})$ & $P$ Value & $\%(95 \% \mathrm{Cl})$ & $P$ Value & $\%(95 \% \mathrm{Cl})$ & $P$ Value ${ }^{c}$ \\
\hline No & $\begin{array}{l}88.0 \\
(86.0-90.1)\end{array}$ & & $\begin{array}{l}70.9 \\
(68.1-73.8)\end{array}$ & & $\begin{array}{l}37.8 \\
(34.8-40.9)\end{array}$ & & $\begin{array}{l}90.7 \\
(88.7-92.7)\end{array}$ & & $8.2(6.5-9.9)$ & \\
\hline \multicolumn{11}{|c|}{ Any cancer (excluding skin cancer) } \\
\hline Yes & $\begin{array}{l}90.9 \\
(80.4-100.0)\end{array}$ & \multirow{2}{*}{$N A^{e}$} & $\begin{array}{l}84.0 \\
(72.9-95.1)\end{array}$ & \multirow{2}{*}{.06} & $\begin{array}{l}25.5 \\
(12.0-39.1)\end{array}$ & \multirow{2}{*}{.10} & 100.0 & \multirow{2}{*}{$N A^{c}$} & $\begin{array}{l}12.8 \\
(0.0-27.2)\end{array}$ & \multirow{2}{*}{$N A^{e}$} \\
\hline No & $\begin{array}{l}88.3 \\
(86.3-90.3)\end{array}$ & & $\begin{array}{l}71.2 \\
(68.3-74.0)\end{array}$ & & $\begin{array}{l}39.0 \\
(35.9-42.0)\end{array}$ & & $\begin{array}{l}90.6 \\
(88.6-92.6)\end{array}$ & & $\begin{array}{l}8.7 \\
(6.9-10.4)\end{array}$ & \\
\hline \multicolumn{11}{|l|}{ Migraine } \\
\hline Yes & $\begin{array}{l}90.9 \\
(84.5-97.4)\end{array}$ & \multirow{2}{*}{.43} & $\begin{array}{l}71.5 \\
(59.4-83.5)\end{array}$ & & $\begin{array}{l}34.6 \\
(23.0-46.2)\end{array}$ & & $\begin{array}{l}95.2 \\
(91.2-99.2)\end{array}$ & & $\begin{array}{l}11.1 \\
(4.4-17.9)\end{array}$ & \multirow{2}{*}{.47} \\
\hline No & $\begin{array}{l}88.2 \\
(86.1-90.3)\end{array}$ & & $\begin{array}{l}71.5 \\
(68.6-74.4)\end{array}$ & .99 & $\begin{array}{l}38.9 \\
(35.8-42.0)\end{array}$ & .49 & $\begin{array}{l}90.5 \\
(88.5-92.6)\end{array}$ & .05 & $\begin{array}{l}8.6 \\
(6.7-10.4)\end{array}$ & \\
\hline \multicolumn{11}{|l|}{ Atrial fibrillation } \\
\hline Yes & $\begin{array}{l}96.7 \\
(92.8-100.0)\end{array}$ & \multirow{2}{*}{$N A^{e}$} & $\begin{array}{l}82.7 \\
(71.9-93.4)\end{array}$ & \multirow{2}{*}{.05} & $\begin{array}{l}57.9 \\
(43.5-72.2) \\
\end{array}$ & \multirow{2}{*}{.01} & \begin{tabular}{|l|}
97.7 \\
$(94.4-100.0)$
\end{tabular} & \multirow{2}{*}{$N A^{c}$} & $\begin{array}{l}22.5 \\
(10.1-34.8) \\
\end{array}$ & \multirow{2}{*}{.04} \\
\hline No & $\begin{array}{l}88.1 \\
(86.0-90.1)\end{array}$ & & $\begin{array}{l}71.1 \\
(68.2-74.0)\end{array}$ & & $\begin{array}{l}37.9 \\
(34.8-41.0)\end{array}$ & & $\begin{array}{l}90.7 \\
(88.7-92.7)\end{array}$ & & $\begin{array}{l}8.3 \\
(6.5-10.1)\end{array}$ & \\
\hline \multicolumn{11}{|l|}{ Depression } \\
\hline Yes & $\begin{array}{l}91.2 \\
(87.0-95.3)\end{array}$ & \multirow{2}{*}{.16} & $\begin{array}{l}79.2 \\
(72.8-85.6) \\
\end{array}$ & \multirow{2}{*}{.01} & $\begin{array}{l}34.1 \\
(27.1-41.1) \\
\end{array}$ & \multirow{2}{*}{.17} & $\begin{array}{l}92.2 \\
(88.1-96.3) \\
\end{array}$ & \multirow{2}{*}{.49} & $\begin{array}{l}10.2 \\
(6.0-14.3) \\
\end{array}$ & \multirow{2}{*}{.47} \\
\hline No & $\begin{array}{l}87.7 \\
(85.5-90.0) \\
\end{array}$ & & \begin{tabular}{|l}
69.8 \\
$(66.7-73.0)$ \\
\end{tabular} & & $\begin{array}{l}39.6 \\
(36.2-42.9) \\
\end{array}$ & & $\begin{array}{l}90.6 \\
(88.4-92.8) \\
\end{array}$ & & $\begin{array}{l}8.5 \\
(6.5-10.4)\end{array}$ & \\
\hline \multicolumn{11}{|c|}{ Number of comorbidities $^{f}$} \\
\hline None & $\begin{array}{l}87.7 \\
(84.1-91.2)\end{array}$ & \multirow{3}{*}{.04} & \begin{tabular}{|l}
70.6 \\
$(65.6-75.6)$ \\
\end{tabular} & \multirow{3}{*}{.002} & $\begin{array}{l}41.6 \\
(36.1-47.1) \\
\end{array}$ & \multirow{3}{*}{.06} & $\begin{array}{l}88.7 \\
(84.7-92.6) \\
\end{array}$ & \multirow{3}{*}{.15} & $\begin{array}{l}7.7 \\
(4.7-10.8) \\
\end{array}$ & \multirow{3}{*}{.29} \\
\hline 1 & $\begin{array}{l}85.9 \\
(82.3-89.6)\end{array}$ & & \begin{tabular}{|l}
66.4 \\
$(61.6-71.1)$ \\
\end{tabular} & & \begin{tabular}{|l}
33.8 \\
$(29.2-38.4)$ \\
\end{tabular} & & $\begin{array}{l}90.9 \\
(87.8-93.9) \\
\end{array}$ & & $\begin{array}{l}7.9 \\
(5.2-10.5)\end{array}$ & \\
\hline$\geq 2$ & $\begin{array}{l}91.8 \\
(88.8-94.9)\end{array}$ & & $\begin{array}{l}78.5 \\
(73.7-83.4)\end{array}$ & & $\begin{array}{l}40.8 \\
(35.1-46.4)\end{array}$ & & $\begin{array}{l}93.4 \\
(90.5-96.3)\end{array}$ & & $\begin{array}{l}11.1 \\
(7.5-14.7)\end{array}$ & \\
\hline
\end{tabular}

Abbreviations: $\mathrm{Cl}$, confidence interval; NA, not applicable.

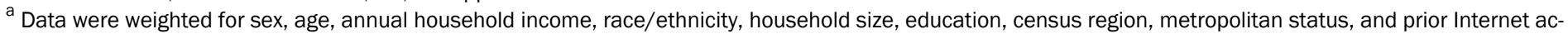

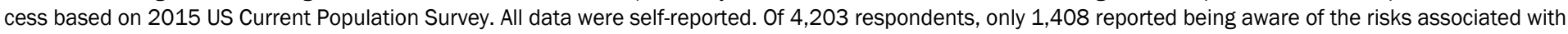
hypertension.

${ }^{b}$ HealthStyles is a web-based panel survey developed and licensed by Porter Novelli Public Services and conducted by GfK's KnowledgePanel.

${ }^{c} P$ values calculated by using Wald $x^{2}$ test.

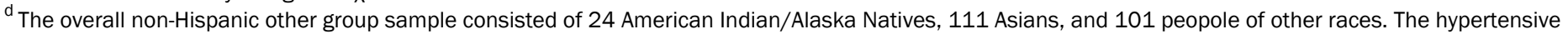

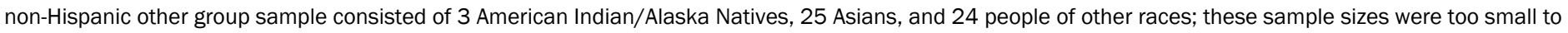
use as individual groups for analyses.

e Sample size was too small for reliable results.

${ }^{\mathrm{f}}$ Comorbidities were heart attack, heart failure, kidney disease, stroke, and dementia. 